\title{
Böses Buch für Zahnärzte
}

Mit Humor ist es so eine Sache. Was dem einen die Lachtränen in die Augen treibt, findet ein anderer zum Gähnen. „Das böse Buch für Zahnärzte“ ist der Versuch dreier Comedy-Autoren, in kurzen Kapiteln Lustiges rund um den Beruf des Zahnarztes zusammenzutragen. Da gibt es von einer „kleinen Geschichte der Zahnmedizin“ über ein „Interview mit Graf Dracula“ bis hin zu den „schönsten Ausreden für den erhöhten Aufwand“ eine ganze Menge Stoff, der zum Schmunzeln anregen soll. Wenn der Leser es am ungelenken Vorwort vorbei geschafft hat und sich nicht am altmodisch anmutenden Layout des Buches stört, kann das auch gelingen. Vielleicht bei Schillers auf Zähne umgedichtetem Werk „Die Glocke“ - nun „Die Extraktion“, oder in der Rubrik „Liebe - wie geht das?“ Dort bekommt der Zahnarzt Tipps, wie er sich beim ersten Date richtig verhält. Ein Beispiel: „Wie sage ich es nach dem ersten Kuss?“ Falsch: „Jetzt gründlich ausspülen, bitte.“

Es gibt im 96 Seiten dicken Buch nicht ganz ernst gemeinte Musik- und Filmtipps für Zahnärzte und natürlich auch einen Leitfaden für Patienten. Einiges, wie die „Sätze, die man von einem Zahnarzt niemals hören wird“, kennt man aus dem Inter- net, vieles ist neu. So erfährt man bei der Lektüre: „Viele Patienten glauben, dass sich die Dr. Best Schwingkopfzahnbürste ausschließlich zum Putzen von Tomaten eignet..."

Wer ein kurzweiliges Büchlein für Zwischendurch sucht und gerne bei Witzen und Cartoons in Zeitungen lacht, liegt mit „Das böse Buch für Zahnärzte“ richtig.

$k p$

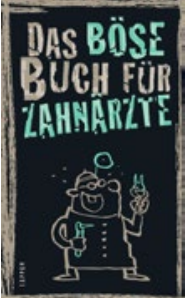

\section{Das böse Buch für Zahnärzte}

Autoren: Linus Höke, Peter Gitzinger,

Roger Schmelzer, Ari Plikat (Illustr.)

9,95 Euro

96 zweifarbige Seiten

Verlag: Lappan

ISBN: 978-3-8303-4336-3

\section{Zahnarztbekenntnisse}

Zugegeben: Für Nicht-Zahnärzte ist der Humor von Dr. Z ein wenig gewöhnungsbedürftig und etwas zu schwarz. Für Zahnärzte allerdings dürfte die leicht sarkastische Note der Daseinsbeschreibung von Dr. $Z$ jedoch durchaus lustig sein. Allein die Beschreibung seiner im Studium erlittenen Traumata, die den Zahnmediziner heute noch im Schlafen wie im Wachen umtreiben, sind ziemlich witzig erzählt. Und man hat ihn direkt vor Augen: Dr. Z, wie er sich als angehender Zahnarzt noch blöder als blöd anstellt und schließlich aufgrund mangelnder Koordination von Instrumenten, Schlauch, Patient, Assistenz und Oberarzt in der benachbarten Box in der Zahnklinik landet.

Heute, nach mehr als 20 Jahren in der Praxis, redet sich Dr. Z seine kleinen und größeren Traumata schön. Inzwischen liegt er auf der Psycho-Couch oder hat zumindest einen Guru gefunden, der ein offenes Ohr für ihn hat. Dr. Z erzählt. Erzählt aus seinem ganz normalen Praxisalltag. Einem Hamsterrad-Alltag, auf den er eigentlich absolut keine Lust mehr hat. Aber er ist halt Zahnarzt. Nicht aus Leidenschaft - nie gewesen, er hat Papis Praxis vor Jahr und Tag übernommen, weil ihm nichts Besseres eingefallen ist - und irgendwer muss sich ja um den ganzen „Mundschrott" kümmern. Und so fantasiert Dr. Z vor sich hin, was er seinen Patienten, Kassen, Kollegen, Ausbildern - kurz allen - gern mal so sagen würde, wenn er denn könnte, wie er wollte. Kurzweilige Rachefantasien, die verstehen lassen, warum Dr. Z seinen wahren Namen nicht preisgeben will.

sas

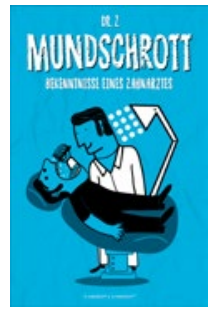

Mundschrott - Bekenntnisse eines Zahnarztes

Autor: Dr. Z

9,99 Euro

219 Seiten

Verlag: Schwarzkopf \& Schwarzkopf

ISBN: 978-3-86265-489-5 